\title{
The Solvability of Mixed Value Problem for the First and Second Approximations of One-Dimensional Nonlinear System of Moment Equations with Microscopic Boundary Conditions
}

\author{
Auzhan Sakabekov $^{1}$. Yerkanat Auzhani ${ }^{1}$ (D)
}

Received: 22 July 2021 / Accepted: 16 December 2021 / Published online: 4 January 2022

(C) The Author(s) 2022

\begin{abstract}
The paper gives a derivation of a new one-dimensional non-stationary nonlinear system of moment equations, that depend on the flight velocity and the surface temperature of an aircraft. Maxwell microscopic condition is approximated for the distribution function on moving boundary, when one fraction of molecules reflected from the surface specular and another fraction diffusely with Maxwell distribution. Moreover, macroscopic boundary conditions for the moment system of equations depend on evenness or oddness of approximation $f_{k}(t, x, c)$, where $f_{k}(t, x, c)$ is partial expansion sum of the molecules distribution function over eigenfunctions of linearized collision operator around local Maxwell distribution. The formulation of initial and boundary value problem for the system of moment equations in the first and second approximations is described. Existence and uniqueness of the solution for the above-mentioned problem using macroscopic boundary conditions in the space of functions $C\left([0, T] ; L^{2}[-a, a]\right)$ are proved.
\end{abstract}

Keywords System of moment equations - Maxwell microscopic condition · Macroscopic boundary conditions $\cdot$ Nonlinear hyperbolic system of equations

\section{Introduction}

Boltzmann equation is used for solving problems of rarefied gas dynamics. Description of a rarefied gas with the help of particles distribution function refers to the transition region between the flows of continuous medium and free-molecular one

Yerkanat Auzhani

erkawww@gmail.com

Auzhan Sakabekov

auzhani@gmail.com

1 Satbayev University, 22a Satpaev Str., 050013 Almaty, Kazakhstan 
and represents sufficiently complicated problem. Prediction of aircraft aerodynamic characteristics at very high altitudes and very high velocities is an important topic in aerospace engineering. They can be determined by the methods of the rarefied gas theory [1]. Interaction of gases with solid surface is the main aspect of gas kinetic theory. Interaction of gas with the surface of a streamlined body plays an important role in high-altitude aerodynamics [2]. When calculating coefficients in formulas for the slip velocity, temperature and concentration near the aircraft surface, it is necessary to take into account the distribution velocity of gas molecules near the wall. In fact, the distribution function of molecules falling on the wall will differ from the distribution function in gas volume, due to collisions with molecules reflected from the wall at a distance from the surface of the order of the free path length of gas molecules (Knudsen layer). Therefore, for the correct description of gas flow near the surface kinetic Boltzmann equation should be used. When calculating aerodynamic characteristics of an aircraft in the high-velocity flow of rarefied gas, it is necessary to supplement Boltzmann equation with the term, that depends on the speed of an aircraft, and the condition on moving boundary has to contain a parameter depending on the surface temperature of an aircraft.

For analysis of aircraft aerodynamic characteristics in transition regime the complete Boltzmann integro-differential equation is used:

$$
\frac{\partial f}{\partial t}+\sum_{i=1}^{3} c_{i} \frac{\partial f}{\partial x_{i}}+\sum_{i=1}^{3} U_{i} \frac{\partial f}{\partial x_{i}}=J(f, f),
$$

where particles distribution function $f=f(t, x, v)$ depends on time, space variable and particle velocity. $c=v-U$ is relative velocity, $U=\left(U_{1}, U_{2}, U_{3}\right)$ is flight velocity of an aircraft, $J(f, f)$ is collision integral. Boltzmann equation is studied with corresponding boundary conditions under which the particles distribution function on the moving surface of a solid body has to meet the conditions. Defining boundary conditions for the surfaces streamlined by rarefied gas is one of the main challenges in kinetic theory.

Boundary conditions for Boltzmann equation connect the distribution functions of falling and reflected molecules. In kinetic theory of gases the most popular model of interaction of molecules with a surface is Maxwell model of specular-diffuse reflection. This model is based on the assumption that one fraction of molecules is reflected specular and another fraction is reflected diffusely. When solving specific problems, Maxwell boundary conditions describe interaction of gas molecules with surface more accurately. In paper [3] it is shown derivation of Maxwell boundary conditions based on the analysis of the most general structure of boundary conditions. A general scheme of boundary conditions generalizing known Cercignani conditions was constructed. In addition, boundary conditions complementing Cercignani conditions were obtained. Both types of boundary conditions allow simulating arbitrary boundary conditions (including specular-diffuse ones) with any degree of accuracy. In paper [4] aerodynamic characteristics of spacecrafts using the method of direct static modeling (the Monte Carlo method) were studied. It was also studied application of described methods to determine aerodynamic characteristics of spacecrafts in a free molecular flow of a rarefied gas. Interaction of 
molecules with the surface is described using various models like Maxwell and Cercignani-Lampis-Lord (CLL). The results of these interactions are calculated using the Monte Carlo method.

Using moment method for the Boltzmann equation results in a system of macroscopic nonlinear partial equations. Using nonlinear system of partial differential equations with appropriate boundary conditions allows to determine flight velocity and surface temperature of aircrafts as well as parameters of the atmosphere. Moment methods differ from each other in the choice of the system of basic functions and the method of determining the expansion coefficients in terms of orthogonal system of functions. In works $[5,6]$ the particles distribution function is presented in the form of a series in three-dimensional Hermite polynomials multiplied by Maxwellian with coefficients depending on the unknown hydrodynamic characteristics of gas. As a sequence, the differential part of obtained system of moment equations contains hydrodynamic characteristics of gas, as coefficients, which complicates the formulation of boundary conditions. In works $[7,8]$ the system of moment equations different from Grad's system was obtained applying the method of polynomial expansion of distribution function in terms of basic function set which represents the product of Sonin polynomials and spherical functions.

In work [9] a new computation algorithm proposed to be an essential part of the moment method to solve Boltzmann equation. The treatment is based on an invariance principle of the collision integral concerning a choice of the basic system of functions over which the distribution function expansion is accomplished. The relations between the matrix elements of an interaction matrix are systematically studied in details. The recurrent relationships between the matrix elements are deduced for the axially symmetric case.

In work [10] approximation of Boltzmann equation based on the moment method was shown. Authors proposed some generalization for setting moment-closure problem from relative entropy to $\varphi \varphi$-divergences, and a corresponding closure based on minimization of $\varphi \varphi$-divergences. The proposed description encapsulates as special cases Grad's classical closure based on expansion in Hermite polynomials and Levermore's entropy-based closure. It is established that the generalization to divergence-based closures enables the construction of extended thermodynamic theories, that avoid essential limitations of the standard moment-closure formulations, such as inadmissibility of the approximate phase-space distribution, potential loss of hyperbolicity and singularity of flux functions at local equilibrium. The divergence-based closure leads to a hierarchy of tractable symmetric hyperbolic systems that retain the fundamental structural properties of Boltzmann equation.

In article [11] the development of continuum models to describe processes in gases in which the particle collisions cannot maintain thermal equilibrium is discussed. Such situations are typically presented in rarefied or diluted gases, for flows in microscopic settings or in general whenever the Knudsen number becomes significant. The continuum models are based on the stochastic description of the gas by Boltzmann equation in the kinetic gas theory. Extended fluid dynamic equations can be derived using moment approximations, such as the regularized 13-moment equations. Moment equations are introduced in detail, and typical results are reviewed for channel flow, cavity flow, and flow past a sphere in the low-Mach number setting, 
for which both evolution equations and boundary conditions are well established. Conversely, nonlinear, high-speed processes require special closures that are still under development. Current approaches are examined along with the challenge of computing the shock wave profiles based on continuum equations.

In paper [12] a globally hyperbolic regularization to the general Grad's moment system in multidimensional spaces is proposed. Systems with moments up to an arbitrary order are studied. The characteristic speeds of the regularized moment system can be analytically given and depend only on the macroscopic velocity and the temperature. The structure of the eigenvalues and eigenvectors of the coefficient matrix is fully clarified. In addition, all characteristic waves are proven to be genuinely nonlinear or linearly degenerate, and the studies on the properties of rarefaction waves, contact discontinuities, and shock waves are included.

In the last two decades the Maximum Entropy Principle (MEP) has been successfully employed to construct macroscopic models, that describe the charge and heat transport in semiconductor devices. These models are obtained starting from Boltzmann transport equations for the charge and the phonon distribution functions, by taking as macroscopic variables the suitable moments of the distributions, and exploiting MEP in order to close the evolution equations for the chosen moments. Important results have also been obtained for the description of charge transport in devices made both of elemental and compound semiconductors, in cases where charge confinement is present and the carrier flow is two- or one-dimensional [13].

Boltzmann equation can be used for modeling rarefied gas flows in transition or kinetic regimes, i.e. to moderate large Knudsen numbers. However, standard moment methods like Grad's approach lack hyperbolicity of the equations. This can lead to instabilities and nonphysical solutions. Based on recent developments in this field in work [14] a quadrature-based moment method leading to globally hyperbolic and rotationally invariant moment equations is derived. Authors present a 1D five moment case of equations and use numerical simulations to compare the new model to standard approaches. Also dedicated numerical methods were tested in solving the new non-conservative moment equations. These first results using the new method show the accuracy of the new method and its benefits compared to Grad's method or other existing models like discrete velocity.

Work [15] defines certain criteria using the characteristic decomposition of the boundary conditions and energy estimates, which is a set of stable boundary conditions for a linear initial boundary value problem, involving a symmetric hyperbolic system. Authors first used these stability criteria to show the instability of Maxwell boundary conditions proposed by Grad [5]. Then a special block structure of the moment equations, which arises due to the recursion relations, and the orthogonality of the Hermite polynomials are recognized. The block structure will help to formulate the stable boundary conditions for an arbitrary order Hermite discretization of Boltzmann equation. The formulation of stable boundary conditions relies on an Onsager matrix, which will be constructed in such a way that the newly proposed boundary conditions stay close to the Maxwell boundary.

In [16] a spatial discretization that preserves the L2-stability by recovering integration-by-parts over the discretized domain and by imposing boundary conditions using a simultaneous-approximation-term (SAT) is presented. Authors 
develop three different forms of the SAT using: (i) characteristic splitting of moment equation boundary conditions; (ii) decoupling of moments in moment equations; and (iii) characteristic splitting of Boltzmann equation boundary conditions. The difference of the first two forms in terms of their usage and implementation is discussed. They show that the third form is equivalent to using an upwind kinetic numerical flux along the boundary, and argue that even though it provides stability, it prescribes the incorrect number of boundary conditions.

Work [17] studies the convergence of stable Hermite approximations (stable Hermite approximations received by the method for posing stable boundary conditions for arbitrary order Hermite approximations in case of linear kinetic equations) and proves the explicit convergence rates under suitable regularity assumptions on the exact solution. The presented convergence rates are confirmed by numerical experiments that involve the linearized BGK equation of rarefied gas dynamics.

Paper [18] aims to improve the convergence of the moment method by introducing filtered hyperbolic moment equations that result in virtually no additional computational overhead, while significantly reducing the error. The filter approach is based on a careful study of averaging solutions of two adjacent moment systems, and the reformulation of the averaging using an artificial collision method, that naturally gives a rise to the filter. Authors study the properties of the filter and show numerical test cases of one-dimensional problems that demonstrate the superior quality of a new filtered moment method.

In this work we study a new system of moment equations which is different from Grad's and Boltzmann's moment systems. The system of moment equations lies in Knudsen intermediate domain and represents a previously unexplored system of partial differential equations of hyperbolic type. Differential part of this system contains two previously unknown parameters depending on time and space variable. Approximation of microscopic boundary condition for Boltzmann equation depends on evenness and oddness of number $k$, where $f_{k}(t, x, c)=f_{0}(\alpha|\mathrm{c}|) \sum_{2 n+l=0}^{k} f_{n l}(t, x) g_{n l}(\alpha \mathrm{c})$. Characteristics of derived hyperbolic system depend on the flight velocity and surface temperature of aircrafts, which are previously unknown. Formulation of boundary conditions for this system is a global problem in rarefied gas dynamics as in case of Grad's moment system. In previous researches we approximated microscopic Maxwell boundary condition at a constant value of parameter $\alpha$ [19]. In current work we consider the case when the particles distribution function is expanded around the local Maxwellian distribution, i.e. $\alpha$ is a variable and depends on time and space variable. For the derived system in Sect. 2 we formulate the initial and boundary value problem in the first and second approximations under Maxwell-Auzhan macroscopic boundary conditions, prove the existence of global, in terms of time, unique solution for the initial and boundary problem for the first approximation of the system of moment equations, and also prove the existence of local, in terms of time, unique solution for the initial and boundary problem for the second approximation of the system of moment equations in space of functions $C\left([0, T] ; L^{2}[-a, a]\right)$. 


\section{On the System of Moment Equations Depending on the Flight Velocity and Surface Temperature of Aircrafts and Approximation of Maxwell Microscopic Condition on Moving Boundary}

Aerodynamic characteristics of streamlined bodies in upper layers of the atmosphere in the transition regime are obtained by calculation. Computational studies of streamlined bodies in the transition regime are carried out on the basis of the kinetic theory of gases. If the initial distribution of gas molecules is known and the condition on the moving boundary is formulated, then further evolution of gas is described by Boltzmann integro-differential equation. The question of gas interaction with surfaces in aerodynamics takes an essential place. The role of laws of molecular particle interaction with surface is manifested the stronger, the more the gas is rarefied. Boundary conditions for Boltzmann equation connect the distribution functions of the falling and reflected molecules. Thus, the problem reduces to solving the mixed problem for Boltzmann equation. The mixed problem for one-dimensional non-stationary Boltzmann equation is approximated by the corresponding problem for the system of moment equations, taking into account the flight velocity of aircrafts under Maxwell microscopic condition on moving boundary. We present a new system of one-dimensional non-stationary nonlinear moment equations, that depend on the flight velocity and the surface temperature of aircrafts as well as approximation of Maxwell microscopic condition on moving boundary. The approximation problem of Maxwell microscopic condition on fixed boundary in case of one-dimensional non-stationary nonlinear Boltzmann equation was solved in [19]. The theorem on the existence of global solution of the mixed problem for 3-dimensional nonlinear Boltzmann equation $(\mathrm{U}=0)$ under Maxwell boundary conditions was proved in [20].

Problem statement. Find a solution of mixed problem for one-dimensional nonlinear non-stationary Boltzmann equation [1]

$$
\begin{gathered}
\frac{\partial f}{\partial t}+|c| \cos \theta \frac{\partial f}{\partial x}+U_{3} \frac{\partial f}{\partial x}=J(f, f), \quad t \in(0, \mathrm{~T}] x \in(-a, a), c \in R_{3}^{c}, \\
\left.f\right|_{t=0}=f^{0}(x, c), \quad(x, c) \in(-a, a) \times R_{3}^{c}, \\
f^{+}(t, x,|c| \cos \theta)=\beta f^{-}(t, x,-|c| \cos \theta)+(1-\beta) \exp \left(-\frac{|c|^{2}}{2 R} \Theta\right), \\
(n, c)=(n,|c| \cos \theta)>0, x=-a \text { or } x=a,
\end{gathered}
$$

where $f \equiv f(t, x, c)$ is particles distribution function in space of velocity and time; $f^{0} \equiv f^{0}(x, c)$ is distribution of particles at initial moment of time (given function); $J(f, f) \equiv \int\left[f\left(c^{\prime}\right) f\left(c_{1}^{\prime}\right)-f(c) f\left(c_{1}\right)\right] \sigma(\cos \chi) d c_{1} d \varepsilon$ is nonlinear collision operator recorded for Maxwell molecules, $J(f, f)$ is the function of t, x, c, $n$ is unit external normal vector of the boundary, $c=v-U, R_{c}^{3}$ is the three dimensional velocity 
space, $\chi$ is the angle between relative velocities of the particles before and after collision.

According to the boundary condition (3) some part of falling particles reflects specular and another part is absorbed into the wall and emits with Maxwell distribution, which corresponds to wall temperature $\Theta \cdot \alpha^{2}=1 /(R \Theta)$ is also a function of time and coordinates. Formula (3) is written under the assumption that boundary (wall or surface) moves with velocity $U_{3} \cdot|c| \cos \theta$ is velocity of the particles falling on the boundary, $-|c| \cos \theta$ is velocity of the particles reflected from the boundary.

Problem (1)-(3) written in the coordinate system is associated with a moving wall, and velocity of movement is a function of time and coordinates, i.e. $U_{3}=U_{3}(t, x)$.

For one-dimensional problems the eigenfunctions of linearized operator have form $[1,21]$ :

$$
g_{n l}(\alpha c)=\gamma_{n l}\left(\frac{\alpha|c|}{\sqrt{2}}\right)^{l} S_{n}^{l+1 / 2}\left(\frac{\alpha^{2}|c|^{2}}{2}\right) P_{l}(\cos \theta), \quad 2 n+l=0,1,2, \ldots,
$$

where $\gamma_{n l}=\sqrt{\frac{\sqrt{\pi} n !(2 l+1)}{2 \Gamma(n+l+3 / 2)}}$ is normalize coefficients, $S_{n}^{l+1 / 2}\left(\frac{\alpha^{2}|c|^{2}}{2}\right)$ is Sonin polynomials, $P_{l}(\cos \theta)$ is Legendre polynomials, $\Gamma$ is Gamma function.

The eigenfunctions $g_{n l}(\alpha c)$ form a full orthogonal system in space of functions $L^{2}\left(R_{c}^{3}\right)$ with weight $f_{0}(\alpha|c|), f_{0}(\alpha|c|)=\left(\alpha^{2} / 2 \pi\right)^{3 / 2} \exp \left(-\alpha^{2} c^{2} / 2\right)$ is local Maxwell distribution. We expand particles distribution function into a series by eigenfunctions $g_{n l}(\alpha c)$ near the local Maxwell distribution

$$
\begin{aligned}
& f(t, x, c)=f_{0}(\alpha|\mathrm{c}|) \sum_{2 n+l=0}^{\infty} f_{n l}(t, x) g_{n l}(\alpha \mathrm{c}), \\
& \text { where } f_{n l}(t, x)=\int_{R_{3}^{c}} f(t, x, c) g_{n l}(\alpha c) d c .
\end{aligned}
$$

For obtaining a system of equations over expansion coefficients we multiply both sides of Eq. (1) by functions $g_{n l}(\alpha c)(2 n+l=0,1,2, \ldots)$ and integrate over the particles velocity

$$
\begin{gathered}
\int_{R_{3}^{c}}\left(\frac{\partial f}{\partial t}+|c| \cos \theta \frac{\partial f}{\partial x}+U_{3} \frac{\partial f}{\partial x}-J(f, f)\right) g_{n l}(\alpha c) d c=0, \\
2 n+l=0,1,2, \ldots,(t, x) \in(0, T] \times(-a, a) .
\end{gathered}
$$

We rewrite equality (6) in form

$$
\begin{aligned}
& \int_{R_{3}^{c}}\left(\frac{\partial f}{\partial t}+|c| \cos \theta \frac{\partial f}{\partial x}+U_{3} \frac{\partial f}{\partial x}-J(f, f)\right) g_{n l}(\alpha c) d c \\
& =\int_{R_{3}^{c}}\left\{\frac{d}{d t}\left(f g_{n l}\right)+\frac{\partial}{\partial x}\left(|c| \cos \theta f g_{n l}\right)-f\left[\frac{d}{d t}\left(g_{n l}\right)+\frac{\partial}{\partial x}\left(|c| \cos \theta g_{n l}\right)\right]-J(f, f) g_{n l}\right\} d c=0,
\end{aligned}
$$


where $\frac{d}{d t}=\frac{\partial}{\partial t}+U_{3} \frac{\partial}{\partial x}$.

Using definition of moments (5) we get

$$
\int_{R_{3}^{c}} \frac{d}{d t}\left(f_{k} g_{n l}\right) d c=\frac{d f_{n l}}{d t} \equiv \frac{\partial f_{n l}}{\partial t}+U_{3} \frac{\partial f_{n l}}{\partial x} .
$$

If in equality (7) instead of $g_{n l}(\alpha c)$ substitute its value and integrate over $R_{3}^{c}$ and using known relations for Sonin and Legendre polynomials [22]

$$
\begin{aligned}
& y S_{n}^{\beta+1}(y)=(n+\beta+1) S_{n}^{\beta}(y)-(n+1) S_{n+1}^{\beta}(y), \\
& S_{n}^{\beta-1}(y)=S_{n}^{\beta}(y)-S_{n-1}^{\beta}(y), \\
& (2 l+1) \mu P_{l}(\mu)=(l+1) P_{l+1}(\mu)-l P_{l-1}(\mu),
\end{aligned}
$$

taking into account equalities (8) and (9) we get the following system of moment equations over coefficients $f_{n l}(t, x)$ (here we omit cumbersome calculations of the coefficients $\left.b_{1}\left(f_{n l}\right), b_{2}\left(f_{n l}\right), b_{3}\left(f_{n l}\right), b_{4}\left(f_{n l}\right)\right)$ :

$$
\begin{aligned}
& \frac{d f_{n l}}{d t}+\frac{\partial}{\partial x}\left[\frac{l}{\alpha \sqrt{(2 l-1)(2 l+1)}}\left(\sqrt{2(n+l+1 / 2)} f_{n, l-1}-\sqrt{2(n+1)} f_{n+1, l-1}\right)\right. \\
& \left.+\frac{l+1}{\alpha \sqrt{(2 l+1)(2 l+3)}}\left(\sqrt{2(n+l+3 / 2)} f_{n, l+1}-\sqrt{2 n} f_{n-1, l+1}\right)\right] \\
& +\frac{d l n \alpha}{d t} b_{1}\left(f_{n l}\right)+\alpha \frac{d U_{3}}{d t} b_{2}\left(f_{n l}\right)+\frac{1}{\alpha} \frac{\partial \ln \alpha}{\partial x}\left[(l+2 n) b_{3}\left(f_{n l}\right)\right. \\
& \left.-2 \sqrt{n(n+l+1 / 2)} b_{3}\left(f_{n-1, l}\right)\right]+\frac{\partial U_{3}}{\partial x} b_{4}\left(f_{n l}\right)=J_{n l}, \\
& 2 n+l=0,1,2, \ldots
\end{aligned}
$$

The coefficients $b_{1}\left(f_{n l}\right), b_{2}\left(f_{n l}\right), b_{3}\left(f_{n l}\right)$ and $b_{4}\left(f_{n l}\right)$ depend linearly on the particles distribution function moments and expressed in the following form

$$
\begin{gathered}
b_{1}\left(f_{n l}\right)=(2 n+l) f_{n l}-2 \sqrt{n(n+l+1 / 2)} f_{n-1, l}, \\
b_{2}\left(f_{n l}\right)=\frac{(l+1) \sqrt{2}}{(2 l+1)(l+2)} \sqrt{\frac{2 l+1}{2 l+3}}\left[-(2 n+l) \sqrt{n} f_{n-1, l+1}+2 \sqrt{n(n-1)(n+l+1 / 2)} f_{n-2, l+1}\right] \\
-\frac{2(2 n+l) \gamma_{n l}}{(2 l+1)(l+2) \alpha^{3}}\left[2 l\left((n+l+1 / 2) I_{n-1, l-1}-n I_{n, l-1}\right)-D_{n l}\right]-\frac{4(n+l+1 / 2) \gamma_{n l}}{(2 l+1)(l+2) \alpha^{3}} \\
\times\left[2 l\left((n+l-1 / 2) I_{n-2, l-1}-(n-1) I_{n-1, l-1}\right)-D_{1, n l}\right],
\end{gathered}
$$

where

$$
I_{n, l-1} \equiv \int_{R_{3}^{c}} f_{0}\left(\frac{\alpha|c|}{\sqrt{2}}\right)^{l-1} S_{n}^{l+1 / 2}\left(\frac{\alpha^{2} c^{2}}{2}\right) P_{l-1}(\mu) f_{k}(t, x, c) d\left(\frac{\alpha|c|}{\sqrt{2}}\right) d \mu d \varphi .
$$


For this integral the following recurrent formula takes place

$$
\begin{aligned}
& I_{n, l-1}=\frac{2(n+l+1 / 2)}{2 n+l+2} I_{n-1, l-1}+\frac{1}{2 n+l+2} D_{1, n l}, \\
& D_{n l}=\sum_{2 N+L=0}^{k} f_{N L}(t, x) \int_{R_{3}^{c}} f_{0}\left(\frac{\alpha|c|}{\sqrt{2}}\right)^{l+1} S_{n}^{l+1 / 2}\left(\frac{\alpha^{2} c^{2}}{2}\right)\left[(l+1) P_{l+1}+l P_{l-1}\right] \\
& \times\left[-(\alpha|c|)^{2} g_{N L}+(L+2 N) g_{N L}-2(N+L+1 / 2) \frac{\gamma_{N L}}{\gamma_{N-1, L}} g_{N-1, L}\right] d\left(\frac{\alpha|c|}{\sqrt{2}}\right) d \mu d \varphi, \\
& D_{1, n l}=\sum_{2 N+L=0}^{k} f_{N L}(t, x) \int_{R_{3}^{c}} f_{0}\left(\frac{\alpha|c|}{\sqrt{2}}\right)^{l+1} S_{n}^{l+1 / 2}\left(\frac{\alpha^{2} c^{2}}{2}\right) P_{l-1}(\cos \theta) \\
& \times\left[-(\alpha|c|)^{2} g_{N L}+(L+2 N) g_{N L}-2(N+L+1 / 2) \frac{\gamma_{N L}}{\gamma_{N-1, L}} g_{N-1, L}\right] d\left(\frac{\alpha|c|}{\sqrt{2}}\right) d \mu d \varphi, \\
& b_{3}\left(f_{n l}\right)=\frac{l}{\sqrt{(2 l-1)(2 l+1)}}\left(\sqrt{2(n+l+1 / 2)} f_{n, l-1}-\sqrt{2(n+1)} f_{n+1, l-1}\right) \\
& +\frac{l+1}{\sqrt{(2 l+1)(2 l+3)}}\left(\sqrt{2(n+l+3 / 2)} f_{n, l+1}-\sqrt{2 n} f_{n-1, l+1}\right) \text {, }
\end{aligned}
$$$$
b_{4}\left(f_{n l}\right)=(2 n+l+1)\left[\left(\frac{2(l+1)(l+2)}{(2 l+1)(2 l+3)(l+3)} \sqrt{\frac{2 l+1}{2 l+5}} \sqrt{n(n+l+3 / 2)} f_{n-1, l+2}\right.\right.
$$$$
\left.-\sqrt{n(n-1)} f_{n-2, l+2}\right)+\frac{1}{2 l+1}\left(\frac{(l+1)^{2}}{2 l+3}+\frac{l^{2}}{2 l-1}\right) f_{n l}+\frac{l(l-1) \gamma_{n l}}{(2 l-1)(2 l+1)} I_{n, l-2}
$$$$
\left.-\frac{(l+1)(l+2) \gamma_{n l}}{(2 l+1)(2 l+3)} \partial_{n, l+2}\right]-2(n+l+1 / 2)\left[\frac{2(l+1)(l+2)}{(2 l+1)(2 l+3)(l+3)} \sqrt{\frac{2 l+1}{2 l+5}}\right.
$$$$
\times\left(\sqrt{n(n-1)} f_{n-2, l+2}-\sqrt{\frac{n(n-1)(n-2)}{n+l+1 / 2}} f_{n-3, l+2}\right)+\frac{1}{2 l+1}\left(\frac{(l+1)^{2}}{2 l+3}+\frac{l^{2}}{2 l-1}\right)
$$$$
\left.\times \sqrt{\frac{n}{n+l+1 / 2}} f_{n-1, l}+\frac{l(l-1) \gamma_{n l}}{(2 l-1)(2 l+1)} I_{n-1, l-2}-\frac{(l+1)(l+2) \gamma_{n l}}{(2 l+1)(2 l+3)} \partial_{n-1, l+2}\right],
$$$$
I_{n, l-2} \equiv \int_{R_{3}^{c}}\left(\frac{\alpha|c|}{\sqrt{2}}\right)^{l} S_{n}^{l+1 / 2}\left(\frac{\alpha^{2} c^{2}}{2}\right) P_{l-2}(\cos \theta) f_{k}(t, x, c) d c
$$$$
I_{n, l-2}=\frac{2(n+l+1 / 2)}{2 n+l+3} I_{n-1, l-2}+\frac{1}{2 n+l+3} \partial_{n, l-2},
$$ 


$$
\begin{aligned}
\partial_{n, l \pm 2} & =\frac{2 \sqrt{2}}{(l+3) \alpha^{3}} \sum_{2 N+L=0}^{k} f_{N L}(t, x) \int_{R_{3}^{c}} f_{0}\left(\frac{\alpha|c|}{\sqrt{2}}\right)^{l+2} S_{n}^{l+1 / 2}\left(\frac{\alpha^{2} c^{2}}{2}\right) P_{l \pm 2}(\mu) \\
\times & {\left[-(\alpha|c|)^{2} g_{N L}+(L+2 N) g_{N L}-2(N+L+1 / 2) \frac{\gamma_{N L}}{\gamma_{N-1, L}} g_{N-1, L}\right] d\left(\frac{\alpha|c|}{\sqrt{2}}\right) d \mu d \varphi . }
\end{aligned}
$$

Moments $J_{n l}$ of the collision integral have form [23]:

$$
J_{n l}=\sum\left\langle N_{3} L_{3} n_{3} l_{3}: l \mid n l 00: l\right\rangle\left\langle N_{3} L_{3} n_{3} l_{3}: l \mid n_{1} l_{1} n_{2} l_{2}: l\right\rangle\left(l_{1} 0 l_{2} 0 / l 0\right)\left(\sigma_{l_{3}}-\sigma_{0}\right) f_{n_{1} l_{1}} f_{n_{2} l_{2}},
$$

where $\left\langle N_{3} L_{3} n_{3} l_{3}: l \mid n_{1} l_{1} n_{2} l_{2}: l\right\rangle$ are generalized Talmi coefficients, $\left(l_{1} 0 l_{2} 0 / l 0\right)$ are Klebsh-Gordon coefficients.

System (10) represents nonlinear hyperbolic system of equations for moments $f_{n l}(t, x)$, since moments of the collision integral are quadratic forms containing $f_{n l}(t, x)$. In addition, differential part of the system contains $U_{3}$ as coefficient (aircraft flight velocity) and $\alpha=\sqrt{1 /(R \Theta)}$, where $\Theta$ is the surface temperature of an aircraft. The time derivative and derivative by spatial variable of flight velocity and of surface temperature of an aircraft are also included into system (10) as coefficients at the lower terms.

System (10) differs from Grad's system since the moments of the distribution function are determined otherwise than Grad's, and structures of these systems are different. Grad decomposed the particles distribution function relative to Hermite polynomials around the local Maxwell function. The decomposition coefficients are determined by formula

$$
a^{N}=\frac{1}{n} \int f H^{(N)}(v) d \xi,
$$

where $\mathrm{v}=\sqrt{\frac{\mathrm{m}}{\mathrm{kT}}} \mathrm{c}=\sqrt{\frac{\mathrm{m}}{\mathrm{kT}}}(\xi-\mathrm{u})$ is relative velocity (relative to the average speed). Expansion coefficients of particles distribution function in terms of Hermite polynomials depend on an unknown parameter $n=\int f \mathrm{~d} \xi$, which represents zero-order moment. In case of system (10) moments $f_{n l}(t, x)$ are determined using equality (5) and $\mathrm{c}=\mathrm{v}-\mathrm{U}_{3}$, where $\mathrm{U}_{3}$ is the flight velocity of an aircraft. Consequently, the expansion coefficients of particles distribution function in terms of Hermite polynomials are different from moments $f_{n l}(t, x)$ (expansion coefficients of $f(t, x, c)$ ) near the local Maxwell distribution for $g_{n l}(\alpha c)$. In addition, structures of Grad's system and moment system (10) are different. If we write differential equation for $\mathrm{a}^{(2)}$ from Grad's system [5] we get

$$
\begin{gathered}
\frac{\partial a_{i j}^{(2)}}{\partial t}+u_{r} \frac{\partial a_{i j}^{(2)}}{\partial x_{r}}+a_{i r}^{(2)} \frac{\partial u_{j}}{\partial x_{r}}+\left(a_{i j}^{(2)}+\delta_{i j}\right) \frac{1}{R T} \frac{d R T}{d t}+\sqrt{R T} \frac{\partial a_{i j r}^{(3)}}{\partial x_{r}} \\
+\frac{\sqrt{R T}}{\rho} a_{i j r}^{(3)} \frac{\partial \rho}{\partial x_{r}}+\frac{3}{2 R T} a_{i j r}^{(3)} \frac{\partial R T}{\partial x_{r}}+\frac{\partial u_{i}}{\partial x_{j}}+\frac{\partial u_{j}}{\partial x_{i}}=J_{i j}^{(2)} .
\end{gathered}
$$


In Eq. (11) $u_{i}, u_{j}, u_{r}$ are the components of gas average speed, $\mathrm{T}$ is the gas temperature, i.e. the coefficients with $a_{i j}^{(2)}, a_{i j r}^{(3)}$ and its derivatives depend on gas macroscopic characteristics. Now we write differential equation for $f_{02}$ from system (10), corresponding to the value of

$$
\begin{gathered}
2 n+l=2 \Rightarrow n=0, l=2 \\
\frac{\partial f_{02}}{\partial t}+U_{3} \frac{\partial f_{02}}{\partial x}+\frac{\partial}{\partial x}\left[\frac{1}{\alpha}\left(\frac{2}{\sqrt{3}} f_{01}+\frac{3}{\sqrt{5}} f_{03}-\frac{2 \sqrt{2}}{\sqrt{15}} f_{11}\right)\right]+2 f_{02} \frac{d \ln \alpha}{d t}-\left(\frac{4}{5 \sqrt{3}} f_{01}+\frac{6 \sqrt{5}}{35} f_{03}\right) \alpha \frac{d U_{3}}{d t} \\
+\left(\frac{4}{\sqrt{3}} f_{01}+\frac{6}{\sqrt{7}} f_{03}-4 \sqrt{\frac{2}{15}} f_{11}\right) \frac{1}{\alpha} \frac{\partial \ln \alpha}{\partial x}+\left(\frac{2 \sqrt{3}}{5} f_{00}+\frac{11}{7} f_{02}-\frac{2 \sqrt{2}}{5} f_{10}\right) \frac{\partial U_{3}}{\partial x}=J_{02} .
\end{gathered}
$$

In Eq. (12) $\mathrm{U}_{3}$ is the flight velocity of an aircraft and $\alpha=\sqrt{1 /(R \Theta)}$, where $\Theta$ is the surface temperature of an aircraft. The derivative of $f_{02}$ relative to $x$ contains $\mathrm{U}_{3}$ as coefficient and derivatives of $f_{01}, f_{03}, f_{11}$ relative to $x$ contain $\frac{1}{\alpha}$ as coefficient. Moreover, the derivatives of $\mathrm{U}_{3}$ and $\alpha$ relative to $t$ and $x$ included into Eq. (12) as coefficients at the lower terms. Consequently, Grad's system and system of moment Eq. (10) are different.

Using corresponding problem for Grad's system of equations we can determine the macroscopic gas characteristics, the flight velocity and surface temperature of an aircraft, if we set the boundary conditions and use mixed problem for system (10). During derivation of system of Boltzmann moment equations [7, 8] the particles distribution function was expanded into eigenfunctions $g_{n l}(\alpha c)$ near the global Maxwell distribution, i.e. at a constant value of $\alpha=\sqrt{1 /(\mathrm{R} \Theta)}$, so the system of Boltzmann moment equations depends on only one parameter. In case of system (10) both quantities $\alpha$ and $\mathrm{U}_{3}$ are functions of time and coordinates. If in (10) parameter $\alpha$ is constant and $\mathrm{U}_{3}=0$, then we obtain Boltzmann system of moment equations [24] as special case of system (10)

$$
\begin{gathered}
\frac{\partial f_{n l}}{\partial t}+\frac{1}{\alpha} \frac{\partial}{\partial x}\left[\frac{l}{\sqrt{(2 l-1)(2 l+1)}}\left(\sqrt{2(n+l+1 / 2)} f_{n, l-1}-\sqrt{2(n+1)} f_{n+1, l-1}\right)\right. \\
\left.+\frac{l+1}{\sqrt{(2 l+1)(2 l+3)}}\left(\sqrt{2(n+l+3 / 2)} f_{n, l+1}-\sqrt{2 n} f_{n-1, l+1}\right)\right]=I_{n l}, \\
2 n+l=0,1, \ldots, k .
\end{gathered}
$$

We multiply both sides of equality (2) by the functions $g_{n l}(\alpha c)(2 n+l=0,1,2, \ldots)$ and integrate over the particles velocity

$$
\begin{gathered}
\int_{R_{3}^{c}}\left[f(0, x, c)-f^{0}(x, c)\right] g_{n l}(\alpha c) d c=0, x \in(-a, a), \\
2 n+l=0,1,2, \ldots,
\end{gathered}
$$


where

$$
\begin{gathered}
f^{0}(x, c)=f_{0}(\alpha|c|) \sum_{2 n+l=0}^{\infty} f_{n l}^{0}(x) g_{n l}(\alpha c) d c, \\
f_{n l}^{0}(x)=\int_{R_{3}^{c}} f^{0}(x, c) g_{n l}(\alpha c) d c .
\end{gathered}
$$

From equality (13) follows initial conditions for the system of Eq. (10) in form

$$
f_{n l}(0, x)=f_{n l}^{0}(x), 2 n+l=0,1,2, \ldots .
$$

An infinite system of Eq. (10) is equivalent to Boltzmann Eq. (1) due to the completeness of the set of eigenfunctions. In practice we study systems with finite number of equations. For a finite system of moment equations we have to formulate macroscopic boundary conditions which were obtained by approximating the microscopic boundary condition for Boltzmann equation. The main idea of moment methods is to set the distribution function more or less arbitrarily-accurate to some macroscopic parameters, which closes the system of moment equations and determine the necessary macroscopic quantities, solving the system of moment equations under the boundary conditions selected in a suitable way.

If in expansion (4) the index $2 n+l$ takes values from 0 to $k$, then we obtain the partial sum

$$
f_{k}(t, x, c)=f_{0}(\alpha|c|) \sum_{2 n+l=0}^{k} f_{n l}(t, x) g_{n l}(\alpha c)
$$

of the series (4). Instead of infinite system of Eq. (10) we consider a system with finite number of moment equations, which corresponds to the partial sum (17). We call this finite system of equations $k$-th approximation of the system of moment equations. Here the problem of closing of a finite system of moment equations arises. The partial sum (17) contains only coefficients $f_{n l}(t, x)$ with non-negative values of the indices $n$ and $l$, therefore, in system (10) corresponding to the $k$-th approximation of the system of moment equations, we set $f_{n l}(t, x)$ equal to zero for negative values of $n$ and $l$. In addition, we nullify coefficients $f_{n l}(t, x)$ for which the indices $n$ and $l$ exceed the value of $k$. For $2 n+l=k(n=0, l=k)$ we set $f_{0, k+1}=0, f_{-1, k+1}=0, f_{-2, k+1}=0$, since (17) does not contain such coefficients.

Boundary conditions for Grad's system of moment equations is a global problem in rarefied gas dynamics. Differential part of system (10) contains two previously unknown parameters depending on time and space variable. Obtaining boundary conditions for finite system of moment equations received from (10) by breaking is also a big challenge as characteristics of the system depend on the flight velocity, surface temperature. Here the same problem of setting boundary conditions as for Grad's system arises.

We set the boundary conditions by approximating microscopic Maxwell condition, which depends on the surface temperature of an aircraft. Note that the 
approximation of boundary condition depends on the evenness or oddness of approximation $k$. The quantity of macroscopic boundary conditions and obtaining them for system of moment equations depends on the parity of approximation number of the moment equation system. More precisely, if $k$ is odd number (even number), then both parts of Maxwell boundary condition multiplies by even (odd) over $l$ eigenfunctions $g_{n, 2 l}(\alpha c)\left(g_{n, 2 l+1}(\alpha c)\right)$ and integrates on velocity semi-space $(n, c)>0$. We approximate boundary condition (3) as follows:

$$
\begin{gathered}
\int_{(n, c)>0}(n, c) f_{2 N+1}^{+}(t, x, c) g_{n, 2 l}(\alpha c) d c-\beta \int_{(n, c)<0}(n, c) f_{2 N+1}^{-}(t, x, c) g_{n, 2 l}(\alpha c) d c \\
-(1-\beta) \int_{(n, c)<0}(n, c) \exp \left(-\frac{|c|^{2}}{2 R \Theta}\right) g_{n, 2 l}(\alpha c) d c=0, \\
2(n+l)=0,2, \ldots, 2 N, x=- \text { a or } x=a,
\end{gathered}
$$

with $k=2 N+1$,

$$
\begin{aligned}
& \int_{(n, c)>0}(n, c) f_{2 N}^{+}(t, x, c) g_{n, 2 l+1}(\alpha c) d c \\
& -\beta \int_{(n, c)<0}(n, c) f_{2 N}^{-}(t, x, c) g_{n, 2 l+1}(-\alpha c) d c \\
& -(1-\beta) \int_{(n, c)<0}(n, c) \exp \left(-\frac{|c|^{2}}{2 R \Theta}\right) g_{n, 2 l+1}(-\alpha c) d c=0, \\
& 2(n+l)+1=1,3, \ldots, 2 N-1, x=-a \text { or } x=a,
\end{aligned}
$$

with $k=2 N$, where $n=(0,0,1)$ with $x=a$ and $n=(0,0,-1)$ with $x=-a$.

In [19] we approximate microscopic Maxwell boundary condition at a constant value of parameter $\alpha$. Boundary conditions for one-dimensional Boltzmann moment equation system and boundary conditions (18) and (19) are similar, but in [19] parameter $\alpha$ is constant and in (18) and (19) this parameter depends on time and space variable. Here we will approximate the microscopic Maxwell condition with variable $\alpha$. Analysis of the number of moment equations and the number of boundary conditions shows that in case $\mathrm{k}=2 N+1(k=2 N)$ a quantity of finite moment system of equations equals to $(k+1)(k+3) / 4\left((k+2)^{2} / 4\right)$ and quantity of macroscopic boundary conditions (18) ((19)) equals to $(k+1)(k+3) / 8(k(k+2) / 4)$ on the left endpoint and the same boundary conditions on the right endpoint of the interval $(-a, a)$. For example, if $k=1$, then a quantity of moment equations in the system equals to 2 and a quantity of macroscopic boundary conditions on the left and on the right endpoints of the interval $(-a, a)$ equals to 1 . When approximating the microscopic boundary condition, we took into account the approximation of Boltzmann equation by moment equations. Thus, the approximation orders for the expansion of the boundary condition and the expansion of the Boltzmann equation are consistent. Macroscopic conditions (18) and (19) we called Maxwell-Auzhan boundary conditions [19]. 


\section{Mixed Value Problems for the First and Second Approximations for the System of Moment Equations with Macroscopic Boundary Conditions}

We will formulate mixed value problem for the finite system of moment equations in the first and second approximations, prove the existence and uniqueness of global, in terms of time, solution for the first approximation, and prove existence of local, in terms of time, solution for the second approximation for the above mentioned problem in functions space $C\left([0, T] ; L^{2}[-a, a]\right)$. To obtain a priori estimation of mixed value problem for non-stationary nonlinear one-dimensional two- and four-moment systems of equations we get integral equalities and then use the spherical representation of vector. Then we obtain initial value problems for the first order ordinary differential equations.

If in (10) $2 n+l$ takes values $(2 n+l=0 \rightarrow n=0, l=0 ; 2 n+l=1 \rightarrow n=0, l=1)$, then we obtain first approximation for the system of moment equations or twomoment system of equations

$$
\begin{aligned}
& \frac{d f_{00}}{d t}+\frac{\partial}{\partial x}\left(\frac{1}{\alpha} f_{01}\right)+\frac{\partial U_{3}}{\partial x}\left(\frac{1}{3} f_{00}\right)=0 \\
& \frac{d f_{01}}{d t}+\frac{\partial}{\partial x}\left(\frac{1}{\alpha} f_{00}\right)+\frac{d \ln \alpha}{d t} f_{01}+\alpha \frac{d U_{3}}{d t}\left(-\frac{1}{3} f_{00}\right)+\frac{1}{\alpha} \frac{\partial \ln \alpha}{\partial x} f_{00}+\frac{\partial U_{3}}{\partial x}\left(\frac{6}{5} f_{01}\right)=0 \\
& \quad t \in(0, \mathrm{~T}], x \in(-a, a),
\end{aligned}
$$

where $f_{00}=f_{00}(t, x)$ and $f_{01}=f_{01}(t, x)$ are unknown functions. For system (20) we set the following initial conditions

$$
f_{00}(0, x)=f_{00}^{0}(x), f_{01}(0, x)=f_{01}^{0}(x), x \in(-a, a),
$$

where $f_{00}^{0}(x), f_{01}^{0}(x)$ are given functions.

For two-moment system of equations we use boundary conditions (18). If in equality (18) expression $2(n+l)$ takes value $0(2(n+l)=0 \Rightarrow n=0, l=0)$, then we obtain each of the boundary conditions at the endpoints of the interval $(-a, a)$ (we omit calculations of boundary integrals)

$$
\begin{aligned}
& \left.\frac{1}{\alpha}\left(f_{01}{ }^{+}-\frac{1}{\sqrt{\pi}} \sqrt{2} f_{00}{ }^{+}\right)\right|_{x=-a}=\left.\beta \frac{1}{\alpha}\left(f_{01}^{-}+\frac{1}{\sqrt{\pi}} \sqrt{2} f_{00}{ }^{-}\right)\right|_{x=-a}+\frac{2 \pi}{\alpha^{4}}(1-\beta), \quad t \in[0, \mathrm{~T}], \\
& \left.\frac{1}{\alpha}\left(f_{01}{ }^{+}+\frac{1}{\sqrt{\pi}} \sqrt{2} f_{00}{ }^{+}\right)\right|_{x=a}=\left.\beta \frac{1}{\alpha}\left(f_{01}^{-}-\frac{1}{\sqrt{\pi}} \sqrt{2} f_{00}^{-}\right)\right|_{x=a}-\frac{2 \pi}{\alpha^{4}}(1-\beta), \quad t \in[0, \mathrm{~T}] .
\end{aligned}
$$

These boundary conditions match with boundary conditions for two-moment system of Boltzmann equations with constant value of parameter $\alpha$ [15], i.e. when the border is motionless. In problem (20)-(23) the boundary moves with velocity $U_{3}$, and parameter $\alpha$ depends on time and coordinates. We have following equalities $f_{00}=\rho, f_{01}=\alpha \rho V$, where $\rho$ is the density of gas, $V$ is the average velocity of gas. 
Thus, it is required to find a solution of the system (20)-(23) and determine aircraft movement velocity and surface temperature.

First we prove correctness of the mixed problem (20)-(23) provided that $\alpha=\alpha(t, x), U_{3}=U_{3}(t, x)$ are given functions.

For problem (20)-(23) the following theorem is valid.

Theorem 1 If initial vector-function $W_{0}=\left(f_{00}^{0}(x), f_{01}^{0}(x)\right)^{\prime}$ belongs to the space of functions $L^{2}[-a, a]$ and $\alpha=\alpha(t, x)>0, U_{3}=U_{3}(t, x)$ are continuous differentiable functions on $[-a, a] \times[0, T]$, then problem (20)-(23) has unique solution $W(t, x)=\left(f_{00}(t, x), f_{01}(t, x)\right)^{\prime}$ in domain $[-a, a] \times[0, T]$ belonging to the space $C\left([0, T] ; L^{2}[-a, a]\right)$, moreover

$$
\|W\|_{C\left([0, T] ; L^{2}[-a, a]\right)} \leq C_{1}\left\|W_{0}\right\|_{L^{2}[-a, a]},
$$

where constant $C_{1}$ does not depend on $W, T$ is any limited positive number.

Proof Let $\mathrm{W}_{0} \in L^{2}[-a, a]$. For establishing an a priori estimation (24) we multiply first equation of system $(20)$ by $f_{00}(t, x)$ and second equation by $f_{01}(t, x)$ and integrate over $[-a, a]$ :

$$
\begin{aligned}
& \int_{-a}^{a}\left\{\frac{1}{2} \frac{d}{d t}\left(f_{00}^{2}+f_{01}^{2}\right)+f_{00} \frac{\partial}{\partial x}\left(\frac{1}{\alpha} f_{01}\right)+f_{01} \frac{\partial}{\partial x}\left(\frac{1}{\alpha} f_{00}\right)+\frac{1}{3} f_{00}^{2} \frac{\partial U_{3}}{\partial x}\right. \\
& \left.+\left[\frac{d \ln \alpha}{d t} f_{01}+\alpha \frac{d U_{3}}{d t}\left(-\frac{1}{3} f_{00}\right)+\frac{1}{\alpha} \frac{\partial \ln \alpha}{\partial x} f_{00}+\frac{\partial U_{3}}{\partial x}\left(\frac{6}{5} f_{01}\right)\right] f_{01}\right\} d x=0
\end{aligned}
$$

We transform the following expressions

$$
\begin{aligned}
\int_{-a}^{a} & \frac{1}{2} \frac{\mathrm{d}}{\mathrm{dt}}\left[f_{00}^{2}+f_{01}^{2}\right] d x=\frac{1}{2} \int_{-a}^{a}\left(\frac{\partial}{\partial t}+U_{3} \frac{\partial}{\partial x}\right)\left[f_{00}^{2}+f_{01}^{2}\right] d x \\
= & \frac{1}{2} \frac{\partial}{\partial t} \int_{-a}^{a}\left[f_{00}^{2}+f_{01}^{2}\right] d x+\frac{1}{2} \int_{-a}^{a} \frac{\partial}{\partial x}\left[U_{3}\left(f_{00}^{2}+f_{01}^{2}\right)\right] d x-\frac{1}{2} \int_{-a}^{a}\left[f_{00}^{2}+f_{01}^{2}\right] \frac{\partial U_{3}}{\partial x} d x \\
= & \frac{1}{2} \frac{\partial}{\partial t} \int_{-a}^{a}\left[f_{00}^{2}+f_{01}^{2}\right] d x-\frac{1}{2} \int_{-a}^{a}\left[f_{00}^{2}+f_{01}^{2}\right] \frac{\partial U_{3}}{\partial x} d x \\
& +\frac{1}{2}\left\{\left.\left[U_{3}\left(f_{00}^{2}+f_{01}^{2}\right)\right]\right|_{x=a}-\left.\left[U_{3}\left(f_{00}^{2}+f_{01}^{2}\right)\right]\right|_{x=-a}\right\} .
\end{aligned}
$$

After integration by parts we get

$$
\begin{aligned}
& \int_{-a}^{a}\left[f_{00} \frac{\partial}{\partial x}\left(\frac{1}{\alpha} f_{01}\right)+f_{01} \frac{\partial}{\partial x}\left(\frac{1}{\alpha} f_{00}\right)\right] d x=\int_{-a}^{a} f_{00} f_{01} \frac{\partial}{\partial x}\left(\frac{1}{\alpha}\right) d x \\
& \quad+\left.\left(\frac{1}{\alpha} f_{00}^{-} f_{01}^{-}\right)\right|_{x=a}-\left.\left(\frac{1}{\alpha} f_{00}^{-} f_{01}^{-}\right)\right|_{x=-a} .
\end{aligned}
$$

Using the boundary conditions (22) and (23) we write equality (27) in the form 


$$
\begin{aligned}
& \int_{-a}^{a}\left[f_{00} \frac{\partial}{\partial x}\left(\frac{1}{\alpha} f_{01}\right)+f_{01} \frac{\partial}{\partial x}\left(\frac{1}{\alpha} f_{00}\right)\right] d x=\int_{-a}^{a} f_{00} f_{01} \frac{\partial}{\partial x}\left(\frac{1}{\alpha}\right) d x \\
& +\left.\left(\frac{1}{\alpha} f_{00}^{-}\left(\sqrt{\frac{2}{\pi}} f_{00}^{-}+\frac{1}{\beta}\left(f_{01}^{+}+\sqrt{\frac{2}{\pi}} f_{00}^{+}\right)+\frac{2 \pi(1-\beta)}{\alpha^{3} \beta}\right)\right)\right|_{x=a} \\
& \quad-\left.\left(\frac{1}{\alpha} f_{00}^{-}\left(-\sqrt{\frac{2}{\pi}} f_{00}^{-}+\frac{1}{\beta}\left(f_{01}^{+}-\sqrt{\frac{2}{\pi}} f_{00}^{+}\right)-\frac{2 \pi(1-\beta)}{\alpha^{3} \beta}\right)\right)\right|_{x=-a} .
\end{aligned}
$$

We substitute the values of integrals from (26) and (28) in equality (25) and rewrite them in the form

$$
\begin{aligned}
\frac{1}{2} & \frac{\partial}{\partial t} \int_{-a}^{a}\left[f_{00}^{2}+f_{01}^{2}\right] d x+\int_{-a}^{a}\left\{-\frac{1}{2}\left[f_{00}^{2}+f_{01}^{2}\right] \frac{\partial U_{3}}{\partial x}+f_{00} f_{01} \frac{\partial}{\partial x}\left(\frac{1}{\alpha}\right)\right. \\
& \left.+\frac{1}{3} f_{00}^{2} \frac{\partial U_{3}}{\partial x}+\frac{d \ln \alpha}{d t} f_{01}^{2}+\alpha \frac{d U_{3}}{d t}\left(-\frac{1}{3} f_{00} f_{01}\right)+\frac{1}{\alpha} \frac{\partial \ln \alpha}{\partial x} f_{00} f_{01}+\frac{\partial U_{3}}{\partial x} \frac{6}{5} f_{01}^{2}\right\} d x \\
& +\frac{1}{2}\left\{\left.\left[U_{3}\left(f_{00}^{2}+f_{01}^{2}\right)\right]\right|_{x=a}-\left.\left[U_{3}\left(f_{00}^{2}+f_{01}^{2}\right)\right]\right|_{x=-a}\right\} \\
& +\left.\frac{1}{\alpha}\left(\sqrt{\frac{2}{\pi}}\left(f_{00}^{-}\right)^{2}+\frac{1}{\beta}\left(f_{01}^{+} f_{00}^{-}+\sqrt{\frac{2}{\pi}} f_{00}^{+} f_{00}^{-}\right)+\frac{2 \pi(1-\beta)}{\alpha^{3} \beta} f_{00}^{-}\right)\right|_{x=a} \\
& +\left.\frac{1}{\alpha}\left(\sqrt{\frac{2}{\pi}}\left(f_{00}^{-}\right)^{2}-\frac{1}{\beta}\left(f_{01}^{+} f_{00}^{-}-\sqrt{\frac{2}{\pi}} f_{00}^{+} f_{00}^{-}\right)+\frac{2 \pi(1-\beta)}{\alpha^{3} \beta} f_{00}^{-}\right)\right|_{x=-a}=0
\end{aligned}
$$

The vector $\mathrm{W}$ is written in the form [25] $\mathrm{W}(t, x)=r(t) \omega(t, x)$, where $\omega(t, x)=\left(\omega_{1}(t, x), \omega_{2}(t, x)\right)^{\prime}, r(t)=\|W(t, .)\|_{L^{2}[-a, a]},\|\omega\|_{L^{2}[-a, a]}=1$.

Substituting the values $f_{00}(t, x)=r(t) \omega_{1}(t, x), f_{01}(t, x)=r(t) \omega_{2}(t, x)$ into (29) we get ordinary differential equation

$$
\frac{d r}{d t}+r P(t)=Q(t)
$$

where

$$
\begin{aligned}
P(t) & =\left.\frac{1}{\alpha}\left(\sqrt{\frac{2}{\pi}}\left(\omega_{1}^{-}\right)^{2}+\frac{1}{\beta}\left(\omega_{2}^{+} \omega_{1}^{-}+\sqrt{\frac{2}{\pi}} \omega_{2}^{+} \omega_{1}^{-}\right)\right)\right|_{x=a} \\
& +\left.\frac{1}{\alpha}\left(\sqrt{\frac{2}{\pi}}\left(\omega_{1}^{-}\right)^{2}-\frac{1}{\beta}\left(\omega_{2}^{+} \omega_{1}^{-}-\sqrt{\frac{2}{\pi}} \omega_{2}^{+} \omega_{1}^{-}\right)\right)\right|_{x=-a}+\frac{U_{3}}{2}\left\{\left.\left[\omega_{1}^{2}+\omega_{2}^{2}\right]\right|_{x=a}-\left.\left[\omega_{1}^{2}+\omega_{2}^{2}\right]\right|_{x=-a}\right\} \\
& +\int_{-a}^{a}\left\{-\frac{1}{2}\left[\omega_{1}^{2}+\omega_{2}^{2}\right] \frac{\partial U_{3}}{\partial x}+\omega_{1} \omega_{2} \frac{\partial}{\partial x}\left(\frac{1}{\alpha}\right)+\frac{1}{3} \omega_{1}^{2} \frac{\partial U_{3}}{\partial x}+\frac{d \ln \alpha}{d t} \omega_{2}^{2}\right. \\
& \left.+\alpha \frac{d U_{3}}{d t}\left(-\frac{1}{3} \omega_{1} \omega_{2}\right)+\frac{1}{\alpha} \frac{\partial \ln \alpha}{\partial x} \omega_{1} \omega_{2}+\frac{\partial U_{3}}{\partial x}\left(\frac{6}{5} \omega_{2}^{2}\right)\right\} d x
\end{aligned}
$$




$$
Q(t)=\left.\left(\frac{2 \pi(1-\beta)}{\alpha^{4} \beta} \omega_{1}^{-}\right)\right|_{x=a}+\left.\left(\frac{2 \pi(1-\beta)}{\alpha^{4} \beta} \omega_{1}^{-}\right)\right|_{x=-a} .
$$

Let's study Eq. (30) with initial condition

$$
r(0)=\left\|W_{0}\right\|=\left\|W_{0}\right\|_{L^{2}[-a, a]} .
$$

The problem (30) and (31) has a solution

$$
r(t)=\exp \left(-\int_{0}^{t} P(\tau) d \tau\right)\left[\|W\|_{0}+\int_{0}^{t} Q(\tau) \exp \left(\int_{0}^{\tau} P(\gamma) d \gamma\right)\right] .
$$

From (32) followed $r(t)$ is bounded for $\forall t: 0<t<T$, where $T$ is arbitrary finite number. Hence $\forall t \in[0, T]$ takes place a priori estimation (24). Existence of solution of problem (20)-(23) may be proved using Galerkin method. Solution uniqueness of problem (20)-(23) followed from estimation (24).

Now we consider mixed problem for a four-moment system of equations which corresponds to the second approximation of (10). If in (10) $2 n+l=1 \rightarrow n=0, l=1 ; 2 n+l=0 \rightarrow n=0, l=0 ; 2 n+l=2 \rightarrow n=0, l=2$ and $n=1, l=0$, then we obtain the second approximation of the moment equation system. Introduce the notations (we omit cumbersome calculations of elements of the matrixes $\left.\mathrm{A}, \mathrm{A}^{\prime}, \mathrm{D}_{1}, \ldots, \mathrm{D}_{8}\right)$ :

$$
\begin{aligned}
& w=f_{01}, u=\left(f_{00}, f_{02}, f_{10}\right)^{\prime}, A=\left(\begin{array}{ll}
1 & 2 / \sqrt{3}-\sqrt{2 / 3}
\end{array}\right), A^{\prime}=\left(\begin{array}{c}
1 \\
2 / \sqrt{3} \\
-\sqrt{2 / 3}
\end{array}\right), \\
& D_{1}=(1), D_{3}=\left(-\frac{1}{\sqrt{3}}-\frac{2}{5 \sqrt{3}} 0\right), D_{5}=\left(1 \frac{2}{\sqrt{5}}-\sqrt{\frac{2}{3}}\right), D_{7}=\left(\frac{6}{5}\right) \text {, } \\
& D_{2}=\left(\begin{array}{ccc}
1 & 0 & 0 \\
0 & 3 & 0 \\
-\sqrt{10} & 0 & 3
\end{array}\right), D_{4}=\left(\begin{array}{c}
0 \\
-4 /(5 \sqrt{3}) \\
0
\end{array}\right), D_{6}=\left(\begin{array}{c}
0 \\
4 / \sqrt{3} \\
-(4+2 \sqrt{3}) / \sqrt{6}
\end{array}\right) \text {, } \\
& D_{8}=\left(\begin{array}{ccc}
1 / 3 & 2 /(5 \sqrt{3}) & 0 \\
2 \sqrt{3} / 5 & 11 / 7 & -2 \sqrt{2} / 5 \\
-\sqrt{2 / 3} & -8 /(5 \sqrt{2}) & 1
\end{array}\right)
\end{aligned}
$$

Then system (10) in second approximation we write in the form 


$$
\begin{aligned}
& \frac{d w}{d t}+\frac{\partial}{\partial x}\left(\frac{1}{\alpha} A u\right)+\frac{d \ln \alpha}{d t} D_{1} w+\alpha \frac{d U_{3}}{d t} D_{3} u+\frac{1}{\alpha} \frac{\partial \ln \alpha}{\partial x} D_{5} u+\frac{\partial U_{3}}{\partial x} D_{7} w=0 \\
& \frac{d u}{d t}+\frac{\partial}{\partial x}\left(\frac{1}{\alpha} A^{\prime} w\right)+\frac{d \ln \alpha}{d t} D_{2} u+\alpha \frac{d U_{3}}{d t} D_{4} w+\frac{1}{\alpha} \frac{\partial \ln \alpha}{\partial x} D_{6} w+\frac{\partial U_{3}}{\partial x} D_{8} u=J(w, u), \\
& \quad t \in(0, \mathrm{~T}], x \in(-a, a),
\end{aligned}
$$

where $J(w, u)=\left(0, J_{02}(w, u), 0\right)^{\prime}, J_{02}(w, u)=\left(\sigma_{2}-\sigma_{0}\right)\left(f_{00} f_{02}-f_{01}^{2} / \sqrt{3}\right) / 2$ is the moment of collision integral.

For system (33) we set the following initial conditions

$$
w(0, x)=w_{0}(x), u(0, x)=u_{0}(x), \quad x \in(-a, a),
$$

where $w_{0}(x)$ is given function and $u_{0}(x)$ is given vector function.

For the second approximation of the moment equations system we use boundary conditions (19). If $k=2$, then $N=1$ and $2(n+l)+1=1 \rightarrow n+l=0 \rightarrow n=l=0$. If in equality (19) we set $n=l=0$ and integrate over semi-space, then at the endpoints of the interval $(-a, a)$ we obtain one each of the boundary conditions (we omit calculations of the boundary integrals)

$$
\begin{aligned}
& {\left.\left[\frac{1}{\alpha}\left(f_{00}^{+}+\frac{2}{\sqrt{3}} f_{02}^{+}-\sqrt{\frac{2}{3}} f_{10}^{+}\right)-\frac{2 \sqrt{2}}{\alpha \sqrt{\pi}} f_{01}^{+}\right]\right|_{x=-a}=\left.\beta\left[\frac{1}{\alpha}\left(f_{00}^{-}+\frac{2}{\sqrt{3}} f_{02}^{-}-\sqrt{\frac{2}{3}} f_{10}^{-}\right)+\frac{2 \sqrt{2}}{\alpha \sqrt{\pi}} f_{01}^{-}\right]\right|_{x=-a}} \\
& +\frac{(1-\beta)}{\alpha^{4}} \frac{\pi}{4 \sqrt{2}} ; \\
& {\left.\left[\frac{1}{\alpha}\left(f_{00}^{+}+\frac{2}{\sqrt{3}} f_{02}^{+}-\sqrt{\frac{2}{3}} f_{10}^{+}\right)+\frac{2 \sqrt{2}}{\alpha \sqrt{\pi}} f_{01}^{+}\right]\right|_{x=a}=\left.\beta\left[\frac{1}{\alpha}\left(f_{00}^{-}+\frac{2}{\sqrt{3}} f_{02}^{-}-\sqrt{\frac{2}{3}} f_{10}^{-}\right)-\frac{2 \sqrt{2}}{\alpha \sqrt{\pi}} f_{01}^{-}\right]\right|_{x=a}} \\
& \quad-\frac{(1-\beta)}{\alpha^{4}} \frac{\pi}{4 \sqrt{2}} .
\end{aligned}
$$

Let $B=\frac{1}{\sqrt{\pi}}(2 \sqrt{2})$ is the matrix and $F=\frac{1}{4 \sqrt{2}}$. We write two last boundary conditions in vector matrix form

$$
\begin{gathered}
\frac{1}{\alpha}\left(A u^{+}-B w^{+}\right)(t,-a)=\beta \frac{1}{\alpha}\left(A u^{-}+B w^{-}\right)(t,-a)+\frac{\pi}{\alpha^{4}}(1-\beta) F, \quad t \epsilon[0, T], \\
\frac{1}{\alpha}\left(A u^{+}+B w^{+}\right)(t, a)=\beta \frac{1}{\alpha}\left(A u^{-}-B w^{-}\right)(t, a)-\frac{\pi}{\alpha^{4}}(1-\beta) F, \quad t \epsilon[0, T] .
\end{gathered}
$$

The eigenvalues of the matrix

$$
\left(\begin{array}{cc}
0 & A \\
A^{\prime} & 0
\end{array}\right)=\left(\begin{array}{cccc}
0 & 1 & 2 / \sqrt{3} & -\sqrt{2 / 3} \\
1 & 0 & 0 & 0 \\
2 / \sqrt{3} & 0 & 0 & 0 \\
-\sqrt{2 / 3} & 0 & 0 & 0
\end{array}\right)
$$


are $\lambda_{1}=-\sqrt{3}, \lambda_{2}=0, \lambda_{3}=0, \lambda_{4}=\sqrt{3}$. Therefore, a system of Eq. (33) with $\alpha$ is constant and $U_{3}=0$ has one arrival characteristic, one departure characteristic and two vertical characteristics. Quantity of boundary conditions for system (33) corresponds to the number of nonzero eigenvalues of the matrix $\left(\begin{array}{cc}0 & A \\ A^{\prime} & 0\end{array}\right)$.

We show that system (33) with initial (34) and boundary (35) and (36) conditions has unique solution in the functions space $C\left([0, T] ; L^{2}[-a, a]\right)$.

For the problem (33)-(36) with $\beta=1$ the following theorem is valid ( $\beta=1$ corresponds to pure mirror reflection from the wall).

Theorem 2 If initial vector-function $W_{0}=\left(w_{0}(x), u_{0}(x)\right)^{\prime}$ belongs to the space of functions $L^{2}[-a, a]$ and $\alpha=\alpha(t, x)>0, U_{3}=U_{3}(t, x)$ are continuous differentiable functions on $[-a, a] \times[0, T]$, then problem (33)-(36) has unique solution $W(t, x)=(w(t, x), u(t, x))^{\prime}$ in domain $[-a, a] \times[0, T]$ belonging to the space $C\left([0, T] ; L^{2}[-a, a]\right)$, moreover

$$
\|W\|_{C\left([0, T] ; L^{2}[-a, a]\right)} \leq C_{1}\left\|W_{0}\right\|_{L^{2}[-a, a]},
$$

where constant $C_{1}$ does not depend on $W$ and $T \sim \mathrm{O}\left(\left\|W_{0}\right\|_{L^{2}[-a, a]}^{-1}\right)$.

For proving this theorem the method of a priori estimation, Galerkin method and Tartar's compensated compactness method [26] are used.

Proof Let $\mathrm{W}_{0} \in L^{2}[-a, a]$. Let's prove estimation (37). We multiple first equation of system (33) by $w$ and second equation by $u$, and integrate from $-a$ to $a$ :

$$
\begin{aligned}
& \int_{-a}^{a}\left\{\frac{1}{2} \frac{\mathrm{d}}{\mathrm{dt}}[(w, w)+(u, u)]+\left[\left(\frac{\partial}{\partial x}\left(\frac{1}{\alpha} \mathrm{Au}\right), w\right)+\left(\frac{\partial}{\partial x}\left(\frac{1}{\alpha} \mathrm{A}^{\prime} \mathrm{w}\right), u\right)\right]\right. \\
& \quad+\frac{d \ln \alpha}{d t}\left[\left(D_{1} w, w\right)+\left(D_{2} u, u\right)\right]+\alpha \frac{d U_{3}}{d t}\left[\left(D_{3} u, w\right)+\left(D_{4} w, u\right)\right] \\
& \left.\quad+\frac{1}{\alpha} \frac{\partial \ln \alpha}{\partial x}\left[\left(D_{5} u, w\right)+\left(D_{6} w, u\right)\right]+\frac{\partial U_{3}}{\partial x}\left[\left(D_{7} w, w\right)+\left(D_{8} u, u\right)\right]\right\} d x=\int_{-a}^{a}(J(w, u), u) d x
\end{aligned}
$$

Then transform following expressions

$$
\begin{aligned}
\int_{-a}^{a} & \frac{1}{2} \frac{\mathrm{d}}{\mathrm{dt}}[(w, w)+(u, u)] d x=\frac{1}{2} \int_{-a}^{a}\left(\frac{\partial}{\partial t}+U_{3} \frac{\partial}{\partial x}\right)[(w, w)+(u, u)] d x \\
= & \frac{1}{2} \frac{\partial}{\partial t} \int_{-a}^{a}[(w, w)+(u, u)] d x+\frac{1}{2} \int_{-a}^{a} \frac{\partial}{\partial x}\left[U_{3}((w, w)+(u, u))\right] d x \\
& -\frac{1}{2} \int_{-a}^{a}[(w, w)+(u, u)] \frac{\partial U_{3}}{\partial x} d x=\frac{1}{2} \frac{\partial}{\partial t} \int_{-a}^{a}[(w, w)+(u, u)] d x-\frac{1}{2} \int_{-a}^{a}[(w, w)+(u, u)] \frac{\partial U_{3}}{\partial x} d x \\
& +\frac{1}{2}\left\{\left.\left[U_{3}((w, w)+(u, u))\right]\right|_{x=a}-\left.\left[U_{3}((w, w)+(u, u))\right]\right|_{x=-a}\right\},
\end{aligned}
$$




$$
\begin{gathered}
\int_{-a}^{a}\left[\left(\frac{\partial}{\partial x}\left(\frac{1}{\alpha} \mathrm{Au}\right), w\right)+\left(\frac{\partial}{\partial x}\left(\frac{1}{\alpha} \mathrm{A}^{\prime} \mathrm{w}\right), u\right)\right] d x=\int_{-a}^{a}\left[\frac{\partial}{\partial x}\left(\frac{1}{\alpha} A u, w\right)+\left(\frac{\partial}{\partial x}\left(\frac{1}{\alpha}\right) A^{\prime} w, u\right)\right] d x \\
=\left[\left(\frac{1}{\alpha} A u^{-}, w^{-}\right)_{x=a}-\left(\frac{1}{\alpha} A u^{-}, w^{-}\right)_{x=-a}\right]+\int_{-a}^{a}\left(\frac{\partial}{\partial x}\left(\frac{1}{\alpha}\right) A^{\prime} w, u\right) d x
\end{gathered}
$$

Taking into account boundary conditions (35) and (36) with $\beta=1$ we rewrite equality (40)

$$
\begin{gathered}
\int_{-a}^{a}\left[\left(\frac{\partial}{\partial x}\left(\frac{1}{\alpha} \mathrm{Au}\right), w\right)+\left(\frac{\partial}{\partial x}\left(\frac{1}{\alpha} \mathrm{A}^{\prime} \mathrm{w}\right), u\right)\right] d x=\left.\left(\frac{1}{\alpha} B w^{-}, w^{-}\right)\right|_{x=a}+\left.\left(\frac{1}{\alpha} B w^{-}, w^{-}\right)\right|_{x=-a} \\
+\left.\frac{1}{\alpha}\left(A u^{+}+B w^{+}, w^{-}\right)\right|_{x=a}-\left.\frac{1}{\alpha}\left(A u^{+}-B w^{+}, w^{-}\right)\right|_{x=-a}+\int_{-a}^{a}\left(\frac{\partial}{\partial x}\left(\frac{1}{\alpha}\right) A^{\prime} w, u\right) d x .
\end{gathered}
$$

Using (39) and (41) we rewrite equality (38) in the following form

$$
\begin{aligned}
& \frac{1}{2} \frac{\partial}{\partial t} \int_{-a}^{a}[(w, w)+(u, u)] d x+\int_{-a}^{a}\left\{\frac{d \ln \alpha}{d t}\left[\left(D_{1} w, w\right)+\left(D_{2} u, u\right)\right]+\alpha \frac{d U_{3}}{d t}\left[\left(D_{3} u, w\right)+\left(D_{4} w, u\right)\right]\right. \\
& \left.+\frac{1}{\alpha} \frac{\partial \ln \alpha}{\partial x}\left[\left(D_{5} u, w\right)+\left(D_{6} w, u\right)\right]+\frac{\partial U_{3}}{\partial x}\left[\left(\left(D_{7}-\frac{1}{2} I\right) w, w\right)+\left(\left(D_{8}-\frac{1}{2} I\right) u, u\right)\right]+\left(\frac{\partial}{\partial x}\left(\frac{1}{\alpha}\right) A^{\prime} w, u\right)\right\} d x \\
& +\frac{U_{3}}{2}\left\{\left.[(u, u)+(w, w)]\right|_{x=a}-\left.[(u, u)+(w, w)]\right|_{x=-a}\right\}+\left.\left(\frac{1}{\alpha} B w^{-}, w^{-}\right)\right|_{x=a}+\left.\left(\frac{1}{\alpha} B w^{-}, w^{-}\right)\right|_{x=-a} \\
& \quad+\left.\frac{1}{\alpha}\left(A u^{+}+B w^{+}, w^{-}\right)\right|_{x=a}-\left.\frac{1}{\alpha}\left(A u^{+}-B w^{+}, w^{-}\right)\right|_{x=-a}=\int_{-a}^{a}(J(w, u), u) d x .
\end{aligned}
$$

I is identity matrix. Let's use spherical representation [25] of vector $\mathrm{W}(t, x)=r(t) \omega(t, x), \quad$ where $\quad(t, x)=\left(\omega_{1}(t, x), \omega_{2}(t, x)\right)^{\prime}, r(t)=\|W(t, .)\|_{L^{2}[-a, a]}$, $\|\omega\|_{L^{2}[-a, a]}=1$.

Substituting the values $w=r(t) \omega_{1}(t, x), u=r(t) \omega_{2}(t, x)$ into (42) we get ordinary equation

$$
\frac{d r}{d t}+r P(t)=r^{2} Q(t)
$$

where

$$
\begin{aligned}
P(t) & =\left.\left(\frac{1}{\alpha} B \omega_{1}^{-}, \omega_{1}^{-}\right)\right|_{x=a}+\left.\left(\frac{1}{\alpha} B{\omega_{1}}^{-}, \omega_{1}^{-}\right)\right|_{x=-a} \\
& +\frac{1}{\alpha}\left[\left(A \omega_{2}^{+}, \omega_{1}^{-}\right)_{x=a}+\left.\left(B{\omega_{1}}^{+}, \omega_{1}^{-}\right)\right|_{x=a}+\left.\left(B \omega_{1}^{+}, \omega_{1}^{-}\right)\right|_{x=-a}-\left.\left(A \omega_{2}^{+}, \omega_{1}^{-}\right)\right|_{x=-a}\right] \\
& +\frac{U_{3}}{2}\left\{\left.\left[\left(\omega_{1}, \omega_{1}\right)+\left(\omega_{2}, \omega_{2}\right)\right]\right|_{x=a}-\left.\left[\left(\omega_{1}, \omega_{1}\right)+\left(\omega_{2}, \omega_{2}\right)\right]\right|_{x=-a}\right\} \\
& +\int_{-a}^{a}\left\{\frac{d \ln \alpha}{d t}\left[\left(D_{1} \omega_{1}, \omega_{1}\right)+\left(D_{2} \omega_{2}, \omega_{2}\right)\right]+\alpha \frac{d U_{3}}{d t}\left[\left(D_{3} \omega_{2}, \omega_{1}\right)+\left(D_{4} \omega_{1}, \omega_{2}\right)\right]\right. \\
& +\frac{1}{\alpha} \frac{\partial \ln \alpha}{\partial x}\left[\left(D_{5} \omega_{2}, \omega_{1}\right)+\left(D_{6} \omega_{1}, \omega_{2}\right)\right] \\
& \left.+\frac{\partial U_{3}}{\partial x}\left[\left(\left(D_{7}-\frac{1}{2} I\right) \omega_{1}, \omega_{1}\right)+\left(\left(D_{8}-\frac{1}{2} I\right) \omega_{2} \omega_{2},\right)\right]+\frac{\partial}{\partial x}\left(\frac{1}{\alpha}\right)\left(\omega_{1}, A \omega_{2}\right)\right\} d x
\end{aligned}
$$




$$
Q(t)=\int_{-a}^{a}\left(J\left(\omega_{1}, \omega_{2}\right), \omega_{2}\right) d x
$$

Let's study Eq. (43) with initial condition

$$
r(0)=\left\|W_{0}\right\|=\left\|W_{0}\right\|_{L^{2}[-a, a]} .
$$

Solution of the problem (43) and (44) has following form

$$
r(t)=\left\{\exp \left(\int_{0}^{t} P(\tau) d \tau\right)\left[\frac{1}{\left\|W_{0}\right\|}-\int_{0}^{t} Q(\tau) \exp \left(-\int_{0}^{\tau} P(\xi) d \xi\right) d \tau\right]\right\}^{-1} .
$$

If $R(t) \equiv \int_{0}^{t} Q(\tau) \exp \left(-\int_{0}^{\tau} P(\xi) d \xi\right) d \tau \leq 0 \forall t$, then $r(t)$ is bounded for $\forall t \in[0,+\infty)$. Let $R(t)>0$. We denote by $T_{1}$ the moment of time when

$$
\frac{1}{\left\|W_{0}\right\|}-\int_{0}^{T_{1}} Q(\tau) \exp \left(-\int_{0}^{\tau} P(\xi) d \xi\right) d \tau=0 .
$$

Then $r(t)$ is bounded for $\forall t \in[0, T]$, where $T<T_{1}$. Moreover $T_{1} \sim \mathrm{O}\left(\|W\|^{-1}\right)$, since integrand $Q(\tau) \exp \left(-\int_{0}^{t} P(\xi) d \xi\right)$ is bounded. Hence $\forall t \in[0, T]$ takes place a priori estimation (37).

Now let's prove the existence of a solution for (33)-(36) using Galerkin method. Let $\left\{v_{l}(x)\right\}_{l=1}^{\infty}$ be a basis in space $L^{2}[-a, a]$, where dimension of vector $v_{l}(x)$ is equal to dimension of vector $W$. For each $m$ we define an approximate solution $W_{m}$ of (33)-(36) as follows:

$$
W_{m}=\sum_{j=1}^{m} c_{j m}(t) v_{j}(x)
$$

$$
\begin{gathered}
\int_{-a}^{a}\left(\frac{\mathrm{dW}}{\mathrm{d} t}+\frac{\partial}{\partial \mathrm{x}}\left(\frac{1}{\alpha} A_{1} W_{m}\right)+\frac{d \ln \alpha}{d t} D_{1,2} W_{m}+\alpha \frac{d U_{3}}{d t} D_{3,4} W_{m}+\frac{1}{\alpha} \frac{\partial \ln \alpha}{\partial x} D_{5,6} W_{m}\right. \\
\left.+\frac{\partial U_{3}}{\partial x} D_{7,8} W_{m}, v_{i}(x)\right) d x=\int_{-a}^{a}\left(J\left(W_{m}\right), v_{i}(x)\right) d x, i=1,2 \ldots m, t \in(0, T] \\
\int_{-a}^{a}\left(\left.\mathrm{~W}_{\mathrm{m}}\right|_{\mathrm{t}=0}-\mathrm{W}_{0 \mathrm{~m}}(x)\right) v_{i}(x) d x=0, \quad i=1,2 \ldots m,
\end{gathered}
$$




$$
\left.\left(\left(\frac{1}{\alpha}\left(A u_{m}^{-} \mp B w_{m}^{-}\right)-\frac{1}{\alpha}\left(A u_{m}^{+} \pm B w_{m}^{+}\right)\right) v_{i}\right)\right|_{x= \pm a}=0, \quad i=1,2 \ldots m, t \epsilon[0, T]
$$

where $W_{0 m}$ is the orthogonal projection of function $W_{0}$ in $L^{2}$ on the subspace, spanned by $v_{1}, \ldots v_{m}$,

$$
\begin{aligned}
& A_{1}=\left(\begin{array}{cc}
0 & A \\
A^{\prime} & 0
\end{array}\right), D_{1,2}=\left(\begin{array}{cc}
D_{1} & 0 \\
0 & D_{2}
\end{array}\right), D_{3,4}=\left(\begin{array}{cc}
0 & D_{3} \\
D_{4} & 0
\end{array}\right), D_{5,6}=\left(\begin{array}{cc}
0 & D_{5} \\
D_{6} & 0
\end{array}\right), D_{7,8}=\left(\begin{array}{cc}
D_{7} & 0 \\
0 & D_{8}
\end{array}\right), \\
& J\left(W_{m}\right)=J\left(w_{m}, u_{m}\right) .
\end{aligned}
$$

We represent $v_{j}(x)$ in form $v_{j}(x)=\left(v_{j}^{(1)}, v_{j}^{(2)}\right)^{\prime}$, where $v_{j}^{(1)}=v_{j 1}, v_{j}^{(2)}=\left(v_{j 2}, v_{j 3}, v_{j 4}\right)^{\prime}$.

The coefficients $c_{j m}(t)$ are determined from the ordinary system of equations

$$
\begin{aligned}
\sum_{j=1}^{m}\left\{\frac{\mathrm{d} c_{\mathrm{jm}}}{\mathrm{dt}} \int_{-a}^{a}\left(v_{j}, v_{i}\right) d x+c_{j m}\left[\left.\left(\frac{1}{\alpha} B v_{j}^{-(1)}, v_{i}^{-(1)}\right)\right|_{x=a}+\left.\left(\frac{1}{\alpha} B v_{j}^{-(1)}, v_{i}^{-(1)}\right)\right|_{x=-a}\right.\right. \\
\quad+\frac{U_{3}}{2}\left(\left.\left(\left(v_{j}^{(1)}, v_{i}^{(1)}\right)+\left(v_{j}^{(2)}, v_{i}^{(2)}\right)\right)\right|_{x=a}-\left.\left(\left(v_{j}^{(1)}, v_{i}^{(1)}\right)+\left(v_{j}^{(2)}, v_{i}^{(2)}\right)\right)\right|_{x=-a}\right) \\
\left.+\left.\quad\left(\frac{1}{\alpha}\left(A v_{j}^{+(2)}+B v_{j}^{+(1)}\right), v_{i}^{-(1)}\right)\right|_{x=a}-\left.\left(\frac{1}{\alpha}\left(A v_{j}^{+(2)}-B v_{j}^{+(1)}\right), v_{i}^{-(1)}\right)\right|_{x=-a}\right] \\
+\int_{-a}^{a}\left(\frac{d \ln \alpha}{d t}\left(\left(D_{1} v_{j}^{(1)}, v_{i}^{(1)}\right)+\left(D_{2} v_{j}^{(2)}, v_{i}^{(2)}\right)\right)+\alpha \frac{d U_{3}}{d t}\left(\left(D_{3} v_{j}^{(2)}, v_{i}^{(1)}\right)+\left(D_{4} v_{j}^{(1)}, v_{i}^{(2)}\right)\right)\right. \\
+\frac{1}{\alpha} \frac{\partial \ln \alpha}{\partial x}\left(\left(D_{5} v_{j}^{(2)}, v_{i}^{(1)}\right)+\left(D_{6} v_{j}^{(1)}, v_{i}^{(2)}\right)\right)+\frac{\partial U_{3}}{\partial x}\left[\left(\left(D_{7}-\frac{1}{2} I\right) v_{j}^{(1)}, v_{i}^{(1)}\right)\right. \\
+\left(\left(D_{8}-\frac{1}{2} I\right) v_{j}^{(2)}, v_{i}^{(2)}\right)-\left(\frac{\partial}{\partial x}\left(\frac{1}{\alpha} A v_{i}^{(2)}\right), v_{j}^{(1)}\right) \\
\left.\left.\quad-\left(\frac{1}{\alpha} A^{\prime} \frac{\partial v_{i}^{(1)}}{\partial x}, v_{j}^{(2)}\right)+\left(\frac{\alpha_{x}^{\prime}}{\alpha^{2}} A v_{j}^{(2)}, v_{i}^{(1)}\right)\right) d x\right\}=\int\left(J\left(\sum_{j=1}^{m} c_{j m} v_{j}\right), v_{i}\right) d x, \\
\quad i=1,2 \ldots m, t \in(0, T],
\end{aligned}
$$

$$
c_{i m}(0)=d_{i m}, \quad i=1,2 \ldots m,
$$

where $d_{i m}$ is $i$-th component of $W_{0 m}$.

We multiply (46) by $c_{i m}(t)$ and sum over $i$ from $l$ to $m$ :

$$
\begin{aligned}
& \int_{-a}^{a}\left(\frac{\mathrm{d} W_{m}}{\mathrm{~d} t}+\frac{\partial}{\partial \mathrm{x}}\left(\frac{1}{\alpha} \mathrm{A}_{1} \mathrm{~W}_{\mathrm{m}}\right)+\frac{d \ln \alpha}{d t} D_{1,2} W_{m}+\alpha \frac{d U_{3}}{d t} D_{3,4} W_{m}+\frac{1}{\alpha} \frac{\partial \ln \alpha}{\partial x} D_{5,6} W_{m}\right. \\
& \left.+\frac{\partial U_{3}}{\partial x} D_{7,8} W_{m}, W_{m}\right) d x=\int_{-a}^{a}\left(\left(J\left(W_{m}\right), W_{m}\right) d x .\right.
\end{aligned}
$$


We proved that $r_{m}(t)$ is bounded in some time interval $\left[0, T_{m}\right]$, where $W_{m}(t, x)=r_{m}(t) \omega_{m}(t, x), T_{m} \approx \mathrm{O}\left(\left\|W_{0 m}\right\|^{-1}\right), T_{m} \geq T \forall m$, and

$$
\left\|W_{m}\right\|_{C\left([0, T] ; L^{2}[-a, a]\right)} \leq C_{2}\left\|W_{0}\right\|_{L^{2}[-a, a]},
$$

where $C_{2}$ is constant and does not depend from $m$.

Solvability of system of Eqs. (45)-(48) (or (49)-(50)) follows from estimation (51).

Thus, the sequence $\left\{W_{m}\right\}$ of approximate solutions of problem (33)-(36) is uniformly bounded in functions space $C\left([0, T] ; L^{2}[-a, a]\right)$. Moreover, homogeneous system of equations $\tau E+\frac{1}{\alpha} A \xi$ with respect to $\tau, \xi$ has only trivial solution. Then it follows from [26] that $U_{m} \stackrel{\alpha}{\rightarrow} U$ is weak in $C\left([0, T] ; L^{2}[-a, a]\right)$ and $J\left(U_{m},\right) \rightarrow J(U)$ is weak in $C\left([0, T] ; L^{2}[-a, a]\right)$ as $m \rightarrow \infty$. Further, it can be shown by standard methods that limit element is a weak solution of the problem (33)-(36).

The theorem is proved.

\section{Conclusion}

The study of the influence of boundary conditions on the aerodynamic characteristics of an aircraft is one of the actual problems in modern aerospace science and practice. This problem especially important for the vehicles flying at high altitudes in modes described by the molecular distribution function. These are the orbital flight of spacecrafts and the movement of aerospace systems in the upper layers of the atmosphere. The system of moment Eq. (10) depends on the flight velocity and the surface temperature of aircrafts and the boundary conditions (18) and (19) depend on the surface temperature of aircrafts. The system (10) is a complex nonlinear hyperbolic system of equations in terms of coefficients of expansion $f(t, x, c)$ to Fourier series. The left side of the system (10) depends on unknown parameters $\alpha$ and $U_{3}$, and the right side is a quadratic form that contains the moments of the particles distribution function. In addition, the boundary conditions (18) and (19) also depend on the unknown parameter $\alpha$. The characteristics of the system of moment equations depend on such unknown parameters as flight velocity and surface temperature of aircrafts, and in general case it is quite difficult to formulate boundary conditions for such systems. Having experience in approximation of Maxwell microscopic condition with constant $\alpha$, we have managed to approximate it with variable $\alpha$. In our work we have formulated boundary conditions depending on the evenness and oddness of the approximation order of $f_{k}(t, x, c)$ in forms (18) and (19).

The proof of correctness for the mixed value problems of the system (10) in various approximations under boundary conditions (18) or (19) is complicated from mathematical point of view. Determination of aerodynamic characteristics such as flight velocity, surface temperature of aircrafts and the atmosphere parameters is important for aerospace engineering. Theorem 1 proves the existence of global, in terms of time, unique solution of the mixed value problem for the first approximation 
of the system of moment Eq. (10) with new macroscopic boundary conditions in space of functions $C\left([0, T] ; L^{2}[-a, a]\right)$. The moments of collision integrals represent undefined quadratic forms, therefore, in Theorem 2 we prove the existence of only a local, in terms of time, solution of the mixed value problem for a four-moment system of Eq. (10). To prove Theorem 2 we use the methods of a priori estimation, Galerkin method and compensated compactness method, which are known tools for proving the existence and uniqueness of solutions of mixed value problems for nonlinear partial derivative equations. Determination of unknown parameters $\alpha$ and $U_{3}$ is an inverse problem for nonlinear hyperbolic system of Eqs. (20) or (33) with initial and boundary conditions (21)-(23) or (34)-(36); this task is a subject for subsequent works.

Acknowledgements The authors would like to thank the anonymous referees for valuable suggestions and comments.

Author Contributions AS: Problem statement, approximation of the Maxwell's boundary condition, formulation of the Theorems 1 and 2, proof. YA: Derivation of the moment system of equations, boundary conditions for two-moment and four-moment system of equations.

Funding This research was funded by Committee of Science of the Ministry of Education and Science of the Republic of Kazakhstan, Grant number AP08856926.

Availability of Data and Material The authors confirm that the data supporting the findings of this study are available within the article [and/or] its supplementary materials.

\section{Declarations}

Conflict of Interest The authors declare that they have no competing interests.

Ethics Approval and Consent to Participate Hereby we confirm that article is not under consideration in other Journals; both authors agree to participate.

Consent for Publication Both authors agree for publication.

Open Access This article is licensed under a Creative Commons Attribution 4.0 International License, which permits use, sharing, adaptation, distribution and reproduction in any medium or format, as long as you give appropriate credit to the original author(s) and the source, provide a link to the Creative Commons licence, and indicate if changes were made. The images or other third party material in this article are included in the article's Creative Commons licence, unless indicated otherwise in a credit line to the material. If material is not included in the article's Creative Commons licence and your intended use is not permitted by statutory regulation or exceeds the permitted use, you will need to obtain permission directly from the copyright holder. To view a copy of this licence, visit http://creativecommons.org/licen ses/by/4.0/.

\section{References}

1. Kogan, M.N.: Dynamic of Rarefied Gas. Nauka, Moscow (1967)

2. Barantcev, R.G.: Interaction of Rarefied Gases with Streamlined Surfaces. Nauka, Moscow (1975)

3. Latyshev, A., Yushkanov, A.: Moment boundary conditions in rarefied gas slip-flow problems. Fluid Dyn. 2, 193-208 (2004) 
4. Khlopkov, Y.I., Zeia, M.M., Khlopkov, A.Y.: Techniques for solving high-altitude tasks in a rarefied gas. Int. J. Appl. Fundam. Res. 1, 156-162 (2014)

5. Grad, G.: Kinetic theory of rarefied gases. Commun. Pure Appl. Math. 2, 331 (1949)

6. Grad, G.: Principles of the Kinetic Theory of Gases. Handbuch der Physik, vol. 3/12, pp. $205-294$. Springer, Berlin (1958)

7. Sakabekov, A.: Initial-boundary value problems for the Boltzmann's moment system equations in an arbitrary approximation. Sb. Russ. Acad. Sci. Math. 77(1), 57-76 (1994)

8. Sakabekov, A.: Initial-Boundary Value Problems for the Boltzmann's Moment System Equations. Gylym, Almaty (2002)

9. Endera, A.I., Enderb, I.A., Lutenkoa, M.B.: Development of the nonlinear moment method for solving the Boltzmann equation in axially symmetric case. Mat. Mod. 14(12), 98-104 (2002)

10. Abdel Malik, M.R.A., van Brummelen, E.H.: Moment closure approximations of the Boltzmann equation based on $\varphi \varphi$-divergences. J. Stat. Phys. 164, 77-104 (2016)

11. Torrilhon, M.: Modeling nonequilibrium gas flow based on moment equations. Annu. Rev. Fluid Mech. 48, 429-458 (2016)

12. Cai, Z., Fan, Y., Li, R.: Globally hyperbolic regularization of Grad's moment system. Commun. Pure Appl. Math. 67(3), 464-518 (2014)

13. Mascali, G., Romano, V.: Exploitation of the maximum entropy principle in mathematical modeling of charge transport in semiconductors. Entropy 19(1), 36 (2017). https://doi.org/10.3390/e19010036

14. Koellermeier, J., Torrilhon, M.: Numerical solution of hyperbolic moment models for the Boltzmann equation. Eur. J. Mech. B Fluids 64, 41-46 (2017)

15. Sarna, N., Torrilhon, M.: On stable wall boundary conditions for the Hermite discretization of the linearised Boltzmann equation. J. Stat. Phys. 170(1), 101-126 (2018)

16. Sarna, N., Kapadia, H., Torrilhon, M.: Simultaneous-approximation-term based boundary discretization for moment equations of rarefied gas dynamics. J. Comput. Phys. 407, 109243 (2020)

17. Sarna, N., Giesselmann, J., Torrilhon, M.: Convergence analysis of Grad's Hermite expansion for linear kinetic equations. SIAM J. Numer. Anal. 58(2), 1164-1194 (2020)

18. Fan, Y., Koellermeier, J.: Accelerating the convergence of the moment method for the Boltzmann equation using filters. J. Sci. Comput. 84, 28 (2020)

19. Sakabekov, A., Auzhani, Y.: Boundary conditions for the one-dimensional nonlinear nonstationary Boltzmann's moment system equations. J. Math. Phys. 55, 123507 (2014)

20. Mischler, S.: Kinetic equations with Maxwell boundary conditions. Annales Scientifique de l'ENS 43(fascicule 5), 719-760 (2010)

21. Cercignani, C.: Theory and Application of the Boltzmann Equation. Scottish Academic Press, Edinburgh and London (1975)

22. Korn, G.A., Korn, T.M.: Mathematical Handbook. McGraw-Hill Book Company, New York, San Francisco, Toronto, London, Sydney (1968)

23. Kumar, K.: Polynomial expansions in Kinetic theory of gases. Ann. Phys. 57, 115-141 (1966)

24. Sakabekov, A.: Mixed value problem for one-dimensional Boltzmann's moment system of equations in odd approximation. Differ. Equ. 28(5), 892-900 (1992). (in Russian)

25. Pokhozhaev, S.I.: On an approach to nonlinear equation. Dokl. Akad. Nauk USSR 247, 1327-1331 (1979)

26. Tartar, L.: Compensated compactness and applications to partial differential equations. In: Knops, R.J. (ed.) Non-Linear Analysis and Mechanics, Heriot-Watt Symposium, vol. IV. Research Notes in Mathematics. vol. 39, pp. 136-212 (1979) 